\title{
INVARIANT DECOMPOSITION OF FUNCTIONS WITH RESPECT TO COMMUTING INVERTIBLE TRANSFORMATIONS
}

\author{
BÁLINT FARKAS, VIKTOR HARANGI, TAMÁS KELETI, \\ AND SZILÁRD GYÖRGY RÉVÉSZ
}

(Communicated by Andreas Seeger)

\begin{abstract}
Consider $a_{1}, \ldots, a_{n} \in \mathbb{R}$ arbitrary elements. We characterize those functions $f: \mathbb{R} \rightarrow \mathbb{R}$ that decompose into the sum of $a_{j}$-periodic functions, i.e., $f=f_{1}+\cdots+f_{n}$ with $\Delta_{a_{j}} f(x):=f\left(x+a_{j}\right)-f(x)=0$. We show that $f$ has such a decomposition if and only if for all partitions $B_{1} \cup B_{2} \cup \cdots \cup B_{N}=\left\{a_{1}, \ldots, a_{n}\right\}$ with $B_{j}$ consisting of commensurable elements with least common multiples $b_{j}$ one has $\Delta_{b_{1}} \ldots \Delta_{b_{N}} f=0$.

Actually, we prove a more general result for periodic decompositions of functions $f: \mathcal{A} \rightarrow \mathbb{R}$ defined on an Abelian group $\mathcal{A}$; in fact, we even consider invariant decompositions of functions $f: A \rightarrow \mathbb{R}$ with respect to commuting, invertible self-mappings of some abstract set $A$.

We also extend our results to functions between torsion free Abelian groups. As a corollary we also obtain that on a torsion free Abelian group the existence of a real-valued periodic decomposition of an integer-valued function implies the existence of an integer-valued periodic decomposition with the same periods.
\end{abstract}

\section{INTRODUCTION}

The starting point of this note is the following observation. If we have $a_{j}$-periodic functions $f_{j}: \mathbb{R} \rightarrow \mathbb{R}, j=1, \ldots, n$, then the sum $f:=f_{1}+f_{2}+\cdots+f_{n}$ satisfies the difference equation

$$
\Delta_{a_{1}} \Delta_{a_{2}} \cdots \Delta_{a_{n}} f=0, \quad \text { where } \quad \Delta_{a_{j}} f(x)=f\left(x+a_{j}\right)-f(x) .
$$

The converse implication, i.e., that the above difference equation would imply existence of a periodic decomposition, however fails already in the simplest situation.

Received by the editors February 2, 2007.

2000 Mathematics Subject Classification. Primary 39A10; Secondary 39B52, 39B72.

Key words and phrases. Periodic functions, periodic decomposition, difference equation, commuting transformations, transformation invariant functions, difference operator, shift operator, decomposition property, Abelian groups, integer-valued functions.

Supported in the framework of the Hungarian-Spanish Scientific and Technological Governmental Cooperation, Project \# E-38/04 and in the framework of the Hungarian-French Scientific and Technological Governmental Cooperation, Project \# F-10/04.

The third author was supported by Hungarian Scientific Foundation grants no. F 43620 and T 49786.

This work was accomplished during the fourth author's stay in Paris under his Marie Curie fellowship, contract \# MEIF-CT-2005-022927. 
For instance, take $a_{1}=a_{2}=a \in \mathbb{R}$ and $f=\mathrm{Id}: \mathbb{R} \rightarrow \mathbb{R}$, the identity. Then $\Delta_{a_{1}} \Delta_{a_{2}} f=0$ holds, but of course $f$ is not a sum of two $a$-periodic functions, as it is not $a$-periodic.

There are two natural ways to overcome this. One might restrict the whole question by requiring that both $f$ and the $f_{j}$ s belong to some function class $\mathcal{F}$, which is then said to have the decomposition property, if the existence of a periodic decomposition in $\mathcal{F}$ is equivalent to the above difference equation (1). For example, it is known that the class $B(\mathbb{R})$ of bounded functions [11], the class $B C(\mathbb{R})$ of bounded continuous functions $[10]$ or the class $U C B(\mathbb{R})$ of uniformly continuous bounded functions [10], [2], or more generally $U C B(\mathcal{A})$ for any locally compact topological group $\mathcal{A}[2]$ do have, while the above example shows that $C(\mathbb{R})$ and $\mathbb{R}^{\mathbb{R}}$ do not have the decomposition property. Some natural questions are still open: for example, it is not known, to the best of our knowledge, whether $B C(\mathcal{A})$ has the decomposition property for any locally compact topological group $\mathcal{A}$. For more about the decomposition property of function classes, see Kadets, Shumyatskiy $[3,4]$, Keleti [5, 6, 7] and Laczkovich, Révész [10, 11].

The other possibility, which is actually our goal now, instead of restricting to a particular function class, is to complement the above difference equation with other conditions of similar type, which then together will be sufficient and necessary for the existence of periodic decompositions with given periods. Suppose that $f$ has a periodic decomposition with periods $a_{1}, \ldots, a_{n}$ and let $B_{1} \cup \cdots \cup B_{N}=\left\{a_{1}, \ldots, a_{n}\right\}$ be a partition such that in each $B_{j}$ the elements are commensurable (that is, they have a common (nonzero integer) multiple) with least common multiple $b_{j}$. Then, by summing up for each $j$ the terms corresponding to the elements in $B_{j}$ we get a periodic decomposition of $f$ with periods $b_{1}, \ldots, b_{N}$. Thus we must have $\Delta_{b_{1}} \Delta_{b_{2}} \cdots \Delta_{b_{N}} f=0$. Therefore we see that if $f$ has a periodic decomposition with periods $a_{1}, \ldots, a_{n}$, then for any partition $B_{1} \cup \cdots \cup B_{N}=\left\{a_{1}, \ldots, a_{n}\right\}$ such that in each $B_{j}$ the elements are commensurable with least common multiple $b_{j}$, we must have $\Delta_{b_{1}} \Delta_{b_{2}} \cdots \Delta_{b_{N}} f=0$. We will show (Corollary 2.7) that this condition is not only necessary but also sufficient.

We note that this characterization easily implies that $f$ has a periodic decomposition (with unprescribed periods) if and only if $\Delta_{b_{1}} \Delta_{b_{2}} \cdots \Delta_{b_{N}} f=0$ for some pairwise incommensurable $b_{1}, \ldots, b_{N}$ and some $N \in \mathbb{N}$. This result was already proved by Mortola and Peirone [12].

In this paper, we will consider a rather general situation: not only translations on $\mathbb{R}$ but mappings on arbitrary nonempty sets. So the precise framework is the following. We take an arbitrary nonempty set $A$ and consider transformations $T: A \rightarrow A$. To such a mapping we also associate a difference operator

$$
\Delta_{T} f:=f \circ T-f
$$

A function is then called $T$-periodic (or $T$-invariant) if $\Delta_{T} f=0$. This terminology is naturally motivated by the case when $A=\mathbb{R}$ and the transformation $T$ is simply a translation by an element $a$ of $\mathbb{R}$, i.e., $T(x):=T_{a}(x):=x+a$ for all $x \in \mathbb{R}$. Note that in this case $T$-periodicity of a function coincides with the usual notion of $a$-periodicity. 
Now consider pairwise commuting transformations $T_{1}, T_{2}, \ldots, T_{n}: A \rightarrow A$. We say that a function $f: A \rightarrow \mathbb{R}$ has a $\left(T_{1}, T_{2}, \ldots, T_{n}\right)$-periodic (or invariant) decomposition if

(2) $f=f_{1}+\cdots+f_{n}$ with $f_{j}$ being $T_{j}$-periodic (i.e., $\Delta_{T_{j}} f_{j}=0$ ) for $j=1, \ldots, n$.

We are looking for necessary and sufficient conditions which ensure that such a periodic decomposition exists. A necessary condition is once again clear as noted at the beginning: if $f=f_{1}+f_{2}+\cdots+f_{n}$ holds with $f_{j}$ being $T_{j}$-periodic for $j=1, \ldots, n$, then using the commutativity of the transformations we obtain

$$
\Delta_{T_{1}} \Delta_{T_{2}} \cdots \Delta_{T_{n}} f=0+0+\cdots+0=0 .
$$

As shown above, if we take $T_{1}=T_{2}=$ translation by $a$ on $\mathbb{R}$, we see that having (3) for some function $f: A \rightarrow \mathbb{R}$, does not suffice for the existence of a periodic decomposition (2). (We remark that the already mentioned result that $B(\mathbb{R})$ has the decomposition property was in fact proved in [11] by showing that the space $B(A)$ of bounded functions on any set $A$ has the decomposition property with respect to arbitrary commuting transformations $T_{1}, T_{2}, \ldots, T_{n}$.)

The decomposition problem for translation operators on $\mathbb{R}$ originates from I. Z. Ruzsa. He showed that the identity function $\operatorname{Id}(x)=x$ can be decomposed into a sum of $a$ - and $b$-periodic functions, whenever $a / b$ is irrational. M. Wierdl [13] extended this by showing that if $a_{1}, \ldots, a_{n} \in \mathbb{R}$ are linearly independent over $\mathbb{Q}$ and a function $f: \mathbb{R} \rightarrow \mathbb{R}$ satisfies (1), then it has a decomposition $f=f_{1}+f_{2}+\cdots+f_{n}$ with $a_{j}$-periodic functions $f_{j}: \mathbb{R} \rightarrow \mathbb{R}, j=1, \ldots, n$.

The periodic decomposition (or invariant decomposition) problem for arbitrary, "abstract" transformations completely without structural restrictions on the underlying set or on the function class was addressed in [1]. There a certain Condition $(*)$ was presented, which was shown to be necessary for the existence of periodic decompositions; moreover, it was also proved to be sufficient for $n=1,2,3$ transformations. Now, we restrict ourselves to the case of invertible transformations. Then the above mentioned Condition $(*)$ of [1] simplifies to Condition 2.2 of the present note, and we obtain both necessity and sufficiency for any number $n \in \mathbb{N}$ of transformations.

We also reformulate the result in a particular case: we investigate periodic, i.e., translation invariant decompositions of functions defined on Abelian groups (Corollaries 2.6 and 2.7).

In Section 4, we extend our result to functions between torsion free Abelian groups (Theorem 4.1). As an immediate corollary of this extension, we obtain a positive answer (Corollary 4.2) to the question whether the existence of a real-valued periodic decomposition of an integer-valued function on $\mathbb{R}$ (or more generally, on a torsion free Abelian group) implies the existence of an integer-valued periodic decomposition with the same periods. This question was asked in [9], where several partial results have been proved, for example that for functions defined on $\mathbb{Z}$ the analogous result holds. Here we also show that this assertion about integer-valued decompositions is not true for functions on an arbitrary Abelian group.

A combination of our results with [8] yields a characterization (Corollary 4.3) of those periods $a_{1}, \ldots, a_{n} \in \mathbb{R}$ for which the existence of a real-valued measurable periodic decomposition of an $\mathbb{R} \rightarrow \mathbb{Z}$ function implies the existence of an integervalued measurable periodic decomposition with the same periods. 


\section{Characterization of existence of Periodic Decompositions}

Let $\mathcal{G}$ be the Abelian group generated by the commuting, invertible transformations $T_{1}, \ldots, T_{n}$ acting on a set $A$, i.e., $\mathcal{G}:=\left\langle T_{1}, T_{2}, \ldots, T_{n}\right\rangle$. For an $x \in A$ we call the set $\{S(x): S \in \mathcal{G}\}$ the orbit of $x$ under $\mathcal{G}$. Such a set is often called an orbit of $\mathcal{G}$ as well, whereas this terminology shall not cause ambiguity. For a transformation $T: A \rightarrow A$ the orbits are understood as the orbits of $\langle T\rangle$.

The following observation helps to simplify the later arguments considerably. Note that $T_{j}$-periodicity, hence the existence of a $\left(T_{1}, T_{2}, \ldots, T_{n}\right)$-periodic decomposition of a function, as well as the validity of conditions involving difference operators (e.g., as in (3)) is decided on the orbits of $\mathcal{G}$. This means that we can always restrict considerations to the orbits of $\mathcal{G}$.

Now, using the following notation, we can formulate the condition characterizing existence of periodic decompositions and we can state our main result.

Notation 2.1. For an Abelian group $\mathcal{H}$ and $B=\left\{b_{1}, \ldots, b_{k}\right\} \subseteq \mathcal{H}$ a nonempty set, we set $[B]:=\bigcap_{i=1}^{k}\left\langle b_{i}\right\rangle$.

Condition 2.2. For all orbits $\mathcal{O}$ of $\mathcal{G}$, for all partitions

$$
B_{1} \cup B_{2} \cup \cdots \cup B_{N}=\left\{\left.T_{1}\right|_{\mathcal{O}},\left.T_{2}\right|_{\mathcal{O}}, \ldots,\left.T_{n}\right|_{\mathcal{O}}\right\}
$$

and any element $S_{j} \in\left[B_{j}\right], j=1, \ldots, N$, we have that

$$
\left.\Delta_{S_{1}} \ldots \Delta_{S_{N}} f\right|_{\mathcal{O}}=0 \text { holds. }
$$

Theorem 2.3. Let $T_{1}, \ldots, T_{n}$ be pairwise commuting invertible transformations on a set $A$. Let $f: A \rightarrow \mathbb{R}$ be any function. Then $f$ has a $\left(T_{1}, T_{2}, \ldots, T_{n}\right)$-periodic decomposition if and only if it satisfies Condition 2.2.

Remark 2.4.

1) Of course, $\left[B_{j}\right]$ is a cyclic group here, and in the above Condition 2.2 it suffices to consider only one of the generators of $\left[B_{j}\right]$ instead of all elements $S_{j} \in\left[B_{j}\right]$. If $\mathcal{G}$ is torsion free, then there is a unique generator $S_{j}$ (up to taking possibly the inverse), so Condition 2.2 simplifies.

2) If $T_{k}=T_{a_{k}}$ are translations on $\mathbb{R}$, and if for some $j$ there are incommensurable elements $a_{k}, a_{m}$ with the corresponding transformations $T_{a_{k}}, T_{a_{m}}$ belonging to $B_{j}$, then $\left[B_{j}\right]=\{\mathrm{Id}\}$. So for such partitions, (4) trivializes; hence it suffices to state Condition 2.2 for partitions $B_{1} \cup B_{2} \cup \cdots \cup B_{N}$ for which the elements belonging to each $B_{j}, j=1, \ldots, N$ are all commensurable. According to the above it also suffices to consider $S_{j}$ to be the translation by the least common multiple $b_{j}$ of the elements in $B_{j}$.

Assume now that the underlying set is a group $A=\mathcal{G}$ and the transformations $T_{j}$ are one-sided, say right, multiplications by elements $a_{j} \in \mathcal{G}$. Then commutativity of the transformations $T_{j}$ is equivalent to commutativity of the generating elements $a_{j}$. Note that in this case the orbits of the group are the left cosets of the subgroup generated by $\left\{a_{1}, \ldots, a_{n}\right\}$ in $\mathcal{G}$. Then each transformation acts on each orbit in the same way, so in Condition 2.2 we do not have to restrict anything to the orbits. Hence Theorem 2.3 gives the following.

Corollary 2.5. Let $\mathcal{G}$ be a group such that $a_{1}, \ldots, a_{n} \in \mathcal{G}$ commute pairwise with each other. Then a function $f: \mathcal{G} \rightarrow \mathbb{R}$ decomposes into a sum of right-a ${ }_{j}$-invariant 
functions, $f=f_{1}+\cdots+f_{n}$, if and only if for all partitions $B_{1} \cup B_{2} \cup \cdots \cup B_{N}=$ $\left\{a_{1}, \ldots, a_{n}\right\}$ and for any element $b_{j} \in\left[B_{j}\right]$ (see Notation 2.1) one has

$$
\Delta_{b_{1}}^{(r)} \ldots \Delta_{b_{N}}^{(r)} f=0
$$

with $\Delta_{a}^{(r)}$ denoting the right difference operator: $\left(\Delta_{a}^{(r)}\right) f(x)=f(x a)-f(x)$.

In the special case when the $T_{j}$ are translations on an Abelian group written additively, the above yields immediately:

Corollary 2.6. Let $\mathcal{A}$ be an (additive) Abelian group and $a_{1}, \ldots, a_{n} \in \mathcal{A}$. $A$ function $f: \mathcal{A} \rightarrow \mathbb{R}$ decomposes into a sum of $a_{j}$-periodic functions, $f=f_{1}+\cdots+$ $f_{n}$, if and only if for all partitions $B_{1} \cup B_{2} \cup \cdots \cup B_{N}=\left\{a_{1}, \ldots, a_{n}\right\}$ and for any element $b_{j} \in\left[B_{j}\right]$ (see Notation 2.1) one has

$$
\Delta_{b_{1}} \ldots \Delta_{b_{N}} f=0 \text {. }
$$

In a torsion free Abelian group $\mathcal{A}$, we call the unique generator $b$ (up to taking possibly the inverse) of the cyclic group $\left\langle b_{1}\right\rangle \cap \cdots \cap\left\langle b_{m}\right\rangle=\left[\left\{b_{1}, b_{2}, \ldots, b_{m}\right\}\right]$ the least common multiple of the elements $b_{1}, b_{2}, \ldots, b_{m} \in \mathcal{A}$. Note that with this terminology we have, for example, that the least common multiple of 1 and $\sqrt{2}$ in the group $(\mathbb{R},+)$ is 0 . This is also the case in general: $a$ and $b$ are by definition incommensurable in $\mathcal{A}$ if $[\{a, b\}]=\{e\}$, i.e., if their least common multiple is the unit element $e \in \mathcal{A}$.

We can now reformulate Theorem 2.3 in this special case as follows (see also Remark 2.4).

Corollary 2.7. Let $\mathcal{A}$ be a torsion free Abelian group and $a_{1}, \ldots, a_{n} \in \mathcal{A}$. $A$ function $f: \mathcal{A} \rightarrow \mathbb{R}$ decomposes into a sum of $a_{j}$-periodic functions, $f=f_{1}+\cdots+$ $f_{n}$, if and only if for all partitions $B_{1} \cup B_{2} \cup \cdots \cup B_{N}=\left\{a_{1}, \ldots, a_{n}\right\}$ and $b_{j}$ being the least common multiple of the elements in $B_{j}$ one has

$$
\Delta_{b_{1}} \ldots \Delta_{b_{N}} f=0 \text {. }
$$

Theorem 2.3 can also be applied if $\mathcal{G}$ is a non-Abelian group and among the transformations there are both left multiplications by certain pairwise commuting elements, and some right multiplications by certain further elements again pairwise commuting among themselves. Indeed, a left and a right multiplication can always be interchanged in view of the associativity law, so this way we get pairwise commuting invertible transformations, and Theorem 2.3 can be applied. However, in this case the orbits are double cosets on which our transformations can act differently, so in this case one cannot simplify Condition 2.2 as in Corollary 2.5.

We also remark that if we look for a continuous decomposition of continuous functions $f: \mathbb{R} \rightarrow \mathbb{R}$, then Condition 2.2 is again insufficient: if $f(x)=x$ and $a_{1} / a_{2} \notin \mathbb{Q}$, then Condition 2.2 is satisfied but $f(x)=x$ is not a sum of two continuous, periodic functions, because it is not bounded. In fact, $f(x)=x$ is not even the sum of two measurable periodic functions (see, e.g., in [11]), so Condition 2.2 is insufficient even for measurable decomposition of continuous functions.

\section{The PROOF OF TheOREM 2.3}

The necessity of Condition 2.2 can already be found in [1, Theorem 4] and is proved there even for not necessarily invertible transformations. We give a straightforward proof for the reader's convenience. 
Proposition 3.1 (Necessity). Suppose that $T_{1}, \ldots, T_{n}$ are pairwise commuting invertible transformations on a set $A$. If $f: A \rightarrow \mathbb{R}$ has a $\left(T_{1}, T_{2}, \ldots, T_{n}\right)$-periodic decomposition, then Condition 2.2 is satisfied.

Remark 3.2. Actually, the proof below yields mutatis mutandis the analogous result for functions $f: A \rightarrow \Gamma$, where $\Gamma$ is an arbitrary Abelian group, written additively.

Proof. We can assume that the group of transformations $\left\langle T_{1}, \ldots, T_{n}\right\rangle$ (generated by $\left.T_{1}, \ldots, T_{n}\right)$ acts on $A$ transitively, i.e., that $A$ is already one orbit under the action of the transformations. Given a partition $B_{1} \cup B_{2} \cup \cdots \cup B_{N}=\left\{T_{1}, T_{2}, \ldots, T_{n}\right\}$ and $S_{j} \in\left[B_{j}\right]$, we have to show $\Delta_{S_{1}} \ldots \Delta_{S_{N}} f=0$.

Note that for $T_{i} \in B_{j}$ the function $f_{i}$ is $S_{j}$-periodic as $S_{j}=T_{i}^{m_{i}}$ for some $m_{i}$ (without loss of generality we can assume $m_{i} \geq 0$; otherwise we could repeat the argument for $S_{j}^{-1}=T_{i}^{-m_{i}}$ ), and

$$
f_{i}(x)=f_{i}\left(T_{i}(x)\right)=f_{i}\left(T_{i}^{2}(x)\right)=\cdots=f_{i}\left(T_{i}^{m_{i}}(x)\right)=f_{i}\left(S_{j}(x)\right) .
$$

So by summing up for each fixed $j$ the functions $f_{i}$ corresponding to those transformations $T_{i}$ that belong to $B_{j}$, we get the functions $g_{j}:=\sum_{T_{i} \in B_{j}} f_{i}$ that are still $S_{j}$-periodic; therefore $f=g_{1}+\cdots+g_{N}$ is an $\left(S_{1}, S_{2}, \ldots, S_{N}\right)$-periodic decomposition of $f$. Hence, as $S_{1}, S_{2}, \ldots, S_{N}$ are pairwise commuting and so are $\Delta_{S_{1}}, \Delta_{S_{2}}, \ldots, \Delta_{S_{N}}$, we obtain that indeed $\Delta_{S_{1}} \Delta_{S_{2}} \ldots \Delta_{S_{N}} f=0$.

To prove the sufficiency of Condition 2.2 we will need the following lemma, which is a slightly modified version of a result from [1], where the same result was proved for real-valued functions and the invertibility of the transformations was not assumed. For the sake of completeness we present the same proof in a compacter form for our case.

Lemma 3.3. Let $T, S$ be commuting invertible transformations of $A$ and let $G$ : $A \rightarrow \Gamma$ be a function with values in the (additive) Abelian group $\Gamma$ and satisfying $\Delta_{T} G=0$. Then there exists a function $g: A \rightarrow \Gamma$ satisfying both $\Delta_{T} g=0$ and $\Delta_{S} g=G$ if and only if

(5) $\sum_{i=0}^{n-1} G\left(S^{i}(x)\right)=0 \quad$ whenever $T^{m}(x)=S^{n}(x)$ for some $m \in \mathbb{Z}, n \in \mathbb{N}, x \in A$.

Proof. Suppose first that there exists a $T$-periodic $g: A \rightarrow \Gamma$ with $\Delta_{S} g=G$, and also that $T^{m}(x)=S^{n}(x)$ for some $m \in \mathbb{Z}, n \in \mathbb{N}$ and $x \in A$. Then

$\sum_{i=0}^{n-1} G\left(S^{i}(x)\right)=\sum_{i=0}^{n-1}\left(g\left(S^{i} S(x)\right)-g\left(S^{i}(x)\right)\right)=g\left(S^{n}(x)\right)-g(x)=g\left(T^{m}(x)\right)-g(x)=0$,

by the $T$-periodicity of $g$. So condition (5) is necessary.

We now prove the sufficiency of this condition. Let us consider the set $\widetilde{A}$ of all orbits of the cyclic group $\langle T\rangle$. Since $G$ is $T$-periodic, it is constant on each orbit $\widetilde{\mathbf{x}} \in \widetilde{A}$, i.e., $G(x)=G\left(x^{\prime}\right)$ if $x, x^{\prime} \in \widetilde{\mathbf{x}}$ (with a small abuse of notation we will write $\widetilde{\mathbf{x}}$ for the orbit of $x)$. So the function $\widetilde{G}: \widetilde{A} \rightarrow \Gamma$ defined by $\widetilde{G}(\widetilde{\mathbf{x}})=G(x)$ is well defined. Because of commutativity the transformation $S$ maps orbits of $\langle T\rangle$ into orbits; hence we can define $\widetilde{S}: \widetilde{A} \rightarrow \widetilde{A}$ by $\widetilde{S}(\widetilde{\mathbf{x}}):=\widetilde{\mathbf{y}}$ with $\widetilde{\mathbf{y}}$ the orbit of $S(x)$. Now 
we pass to $\widetilde{A}$, and notice that (5) implies

$$
\sum_{i=0}^{n-1} \widetilde{G}\left(\widetilde{S}^{i}(\widetilde{\mathbf{x}})\right)=0 \quad \text { whenever } \widetilde{S}^{n}(\widetilde{\mathbf{x}})=\widetilde{\mathbf{x}} \text { for some } n \in \mathbb{N} \text { and } \widetilde{\mathbf{x}} \in \widetilde{A} \text {. }
$$

Consider the orbits of $\langle\widetilde{S}\rangle$ in $\widetilde{A}$. By the axiom of choice we select for each such orbit $\beta \subset \widetilde{A}$ an element $\widetilde{\mathbf{x}}_{\beta} \in \beta$. We claim that the function defined as follows (understanding empty sums as 0 ) is well defined:

$$
\widetilde{g}(\widetilde{\mathbf{x}}):= \begin{cases}\widetilde{G}\left(\widetilde{\mathbf{x}}_{\beta}\right)-\sum_{i=0}^{n-1} \widetilde{G}\left(\widetilde{S}^{i}(\widetilde{\mathbf{x}})\right), & \text { if } \widetilde{\mathbf{x}} \in \beta \text { and } \widetilde{S}^{n}(\widetilde{\mathbf{x}})=\widetilde{\mathbf{x}}_{\beta} \text { with } n \geq 0, \\ \widetilde{G}\left(\widetilde{\mathbf{x}}_{\beta}\right)+\sum_{i=n}^{-1} \widetilde{G}\left(\widetilde{S}^{i}(\widetilde{\mathbf{x}})\right), & \text { if } \widetilde{\mathbf{x}} \in \beta \text { and } \widetilde{S}^{n}(\widetilde{\mathbf{x}})=\widetilde{\mathbf{x}}_{\beta} \text { with } n<0 .\end{cases}
$$

Indeed, if both $\widetilde{S}^{n}(\widetilde{\mathbf{x}})=\widetilde{\mathbf{x}}_{\beta}$ and $\widetilde{S}^{m}(\widetilde{\mathbf{x}})=\widetilde{\mathbf{x}}_{\beta}$ hold with, say, $n-m>0$, then $\widetilde{S}^{n-m} \widetilde{S}^{m}(\widetilde{\mathbf{x}})=\widetilde{S}^{m}(\widetilde{\mathbf{x}})$, so (6) yields that the difference of the two different expressions that define $\widetilde{g}(\widetilde{\mathbf{x}})$ is

$$
\sum_{i=m}^{n-1} \widetilde{G}\left(\widetilde{S}^{i}(\widetilde{\mathbf{x}})\right)=\sum_{i=0}^{n-m-1} \widetilde{G}\left(\widetilde{S}^{i} \widetilde{S}^{m}(\widetilde{\mathbf{x}})\right)=0 .
$$

For $\widetilde{S}^{n}(\widetilde{\mathbf{x}})=\widetilde{\mathbf{x}}_{\beta}, n>0$, we have $\widetilde{S}^{n-1} \widetilde{S}(\widetilde{\mathbf{x}})=\widetilde{\mathbf{x}}_{\beta}$ and hence, by definition of $\widetilde{g}$,

$$
\begin{aligned}
\Delta_{\widetilde{S}} \widetilde{g}(\widetilde{\mathbf{x}}) & =\widetilde{g}(\widetilde{S}(\widetilde{\mathbf{x}}))-\widetilde{g}(\widetilde{\mathbf{x}}) \\
& =\left(\widetilde{G}\left(\widetilde{\mathbf{x}}_{\beta}\right)-\sum_{i=0}^{n-2} \widetilde{G}\left(\widetilde{S^{i}} \widetilde{S}(\widetilde{\mathbf{x}})\right)\right)-\left(\widetilde{G}\left(\widetilde{\mathbf{x}}_{\beta}\right)-\sum_{i=0}^{n-1} \widetilde{G}\left(\widetilde{S}^{i}(\widetilde{\mathbf{x}})\right)\right)=\widetilde{G}(\widetilde{\mathbf{x}}) .
\end{aligned}
$$

It follows similarly that $\Delta_{\widetilde{S}} \widetilde{g}(\widetilde{\mathbf{x}})=\widetilde{G}(\widetilde{\mathbf{x}})$ also in the cases $n=0, n<0$.

Now we pull back $\widetilde{g}: \widetilde{A} \rightarrow \widetilde{A}$ to $A$; that is, we set $g(x):=\widetilde{g}(\widetilde{\mathbf{x}})$, where $\widetilde{\mathbf{x}}$ is the orbit of $x$ under $\langle T\rangle$. It is straightforward that $\Delta_{T} g=0$ and $\Delta_{S} g=G$.

We complete the proof of Theorem 2.3 by proving the sufficiency of Condition 2.2.

Remark 3.4. The reader will have no difficulty in verifying that the proof below yields also the following assertions.

1) Theorem 2.3 remains valid for functions taking values in an arbitrary divisible torsion free group (which can be written in the form $\bigoplus_{I} \mathbb{Q}=\mathbb{Q}^{I}$ for some index set $I)$.

2) There is a positive integer $M=M\left(T_{1}, \ldots, T_{n}\right)$ such that, whenever $f$ takes its values in an additive subgroup $\Gamma$ of $\mathbb{Q}^{I}$, then the functions in the periodic decomposition can be chosen to have values $\frac{x}{M}$ with $x \in \Gamma$.

3) If $T_{1}$ is of infinite order, $T_{1}^{m_{1}}=T_{2}^{m_{2}}=\ldots=T_{k}^{m_{k}}\left(m_{1} \in \mathbb{N}, m_{2} \ldots, m_{k} \in \mathbb{Z} \backslash\{0\}\right)$ and $T_{k+1}, \ldots, T_{n}$ are unrelated to $T_{1}$ (see the definition below), then as the constant $M\left(T_{1}, T_{2}, \ldots, T_{n}\right)$ we can take $m_{1} M\left(T_{2}, \ldots, T_{n}\right) M\left(T_{k+1}, \ldots, T_{n}\right)$.

Proof of the sufficiency of Condition 2.2 in Theorem 2.3. Our proof is by induction on the number $n \in \mathbb{N}$ of transformations. The case $n=1$ is obvious.

As pointed out before, we assume without loss of generality that $\mathcal{G}:=\left\langle T_{1}, \ldots, T_{n}\right\rangle$ acts transitively on $A$, i.e., $A$ is one orbit of $\mathcal{G}$. Following [1] we say that the transformations $T_{i}$ and $T_{j}$ are related, if there are $m, k \in \mathbb{Z} \backslash\{0\}$ with $T_{i}^{m}=T_{j}^{k}$. This is clearly an equivalence relation. 
If possible, let us take as $T_{1}$ an element of infinite order from $\left\{T_{1}, T_{2}, \ldots, T_{n}\right\}$. Let us also assume for notational convenience that $\left\{T_{1}, T_{2}, \ldots, T_{k}\right\}, k \leq n$, are exactly the elements among the $T_{j}$ s being related to $T_{1}$. This means in particular that there exist $m_{j} \in \mathbb{Z} \backslash\{0\}, m_{1} \in \mathbb{N}$ such that $T_{j}^{m_{j}}=T_{1}^{m_{1}}=: U_{1}$ for all $j=1, \ldots, k$, and the other elements $\left\{T_{k+1}, \ldots, T_{n}\right\}$ are then all unrelated to $T_{j}, j=1, \ldots, k$. Note that if all the elements $T_{1}, T_{2}, \ldots, T_{n}$ have finite order, then they are also all related, so $n=k$.

We define the functions $g:=\Delta_{T_{1}} f, h:=\Delta_{U_{1}} f$. In case $U_{1}=T_{1}$, these two functions coincide, but the following arguments still remain valid. It might also happen that $U_{1}=\mathrm{Id}$, in this case $h=0$, but neither does this affect the validity of the following.

We will apply the induction hypothesis to $g$ and $h$. For this purpose we first check that Condition 2.2 is satisfied for the function $g$ and the transformations $\left\{T_{2}, T_{3}, \ldots, T_{n}\right\}$ and for the function $h$ and the transformations $\left\{T_{k+1}, \ldots, T_{n}\right\}$, respectively. By assumption, we have Condition 2.2 for $f$ and the transformations $\left\{T_{1}, T_{2}, \ldots, T_{n}\right\}$; we are to apply this by choosing the partitions $B_{1} \cup B_{2} \cup \cdots \cup B_{N}$ in a particular way.

Considering partitions of $\left\{T_{1}, T_{2}, \ldots, T_{n}\right\}$ with $B_{1}:=\left\{T_{1}\right\}$ and the other blocks being arbitrary and taking $S_{1}=T_{1} \in\left[B_{1}\right]$ and $S_{j} \in\left[B_{j}\right]$ arbitrary, we see that Condition 2.2 is satisfied for $g$ and the transformations $\left\{T_{2}, T_{3}, \ldots, T_{n}\right\}$. Similarly, if we consider $B_{1}=\left\{T_{1}, T_{2}, \ldots, T_{k}\right\}, S_{1}=U_{1}$ and the other blocks arbitrary, we see that $h$ satisfies Condition 2.2 with the transformations $\left\{T_{k+1}, T_{k+2}, \ldots, T_{n}\right\}$.

Now the inductive hypothesis yields the two decompositions

$$
\begin{aligned}
g & =g_{2}+\cdots+g_{k}+g_{k+1}+\cdots+g_{n}, & & \text { with } \Delta_{T_{j}} g_{j}=0, \quad 2 \leq j \leq n, \\
\text { and } & h & =h_{k+1}+\cdots+h_{n}, &
\end{aligned}
$$

(Note again that, if incidentally $k=n$, then $h=0$ by assumption.) For all $j=2, \ldots, n$ we define the function

$$
G_{j}(x):=\frac{1}{m_{1}} \sum_{\mu=0}^{m_{1}-1} g_{j}\left(T_{1}^{\mu}(x)\right)
$$

which is, of course, $T_{j}$-periodic. Moreover, for $j=2, \ldots, k$ we can even claim $\Delta_{T_{1}} G_{j}=0$. Indeed, one has

$$
\Delta_{T_{1}} G_{j}(x)=G_{j}\left(T_{1}(x)\right)-G_{j}(x)=\frac{1}{m_{1}}\left(g_{j}\left(T_{1}^{m_{1}}(x)\right)-g_{j}(x)\right)=0,
$$

because $T_{1}^{m_{1}}=T_{j}^{m_{j}}$ and $g_{j}$ is $T_{j}$-periodic. By definition we can write

$$
h(x)=f\left(T_{1}^{m_{1}}(x)\right)-f(x)=\sum_{\mu=0}^{m_{1}-1} g\left(T_{1}^{\mu}(x)\right)=\sum_{\mu=0}^{m_{1}-1} \sum_{j=2}^{n} g_{j}\left(T_{1}^{\mu}(x)\right)=m_{1} \sum_{j=2}^{n} G_{j}(x),
$$

whence the decomposition of $h$ entails

$$
\frac{1}{m_{1}} \sum_{j=k+1}^{n} h_{j}(x)=\sum_{j=2}^{n} G_{j}(x) .
$$


Now, the functions $F_{j}, j=2, \ldots, n$, defined by

$$
F_{j}:= \begin{cases}g_{j}-G_{j}, & 2 \leq j \leq k \\ g_{j}-G_{j}+\frac{1}{m_{1}} h_{j}, & k+1 \leq j \leq n\end{cases}
$$

are undoubtedly $T_{j}$-periodic. According to $(7)$ we still have

$$
\sum_{j=2}^{n} F_{j}=\sum_{j=2}^{n} g_{j}=g=\Delta_{T_{1}} f
$$

Now we prove that we can apply Lemma 3.3 with $S=T_{1}$ and $T=T_{j}$ to all functions $F_{j}$. For the indices $j \geq k+1$ these transformations are unrelated to $T_{1}$. This means that $T_{1}^{m}=T_{j}^{m^{\prime}}$ cannot hold for $m, m^{\prime} \in \mathbb{Z} \backslash\{0\}$. Nor is it possible that $T_{1}^{m}=\operatorname{Id}=T_{j}^{0}$ with $m \in \mathbb{Z} \backslash\{0\}$, because $T_{1}$ was chosen to be of infinite order. (Should this choice be impossible, then all elements are related, i.e., $n=k$ and this case is empty.) So we see that for $j \geq k+1$ condition (5) of Lemma 3.3 is void, whence the existence of a "lift-up" $f_{j}$ with $\Delta_{T_{1}} f_{j}=F_{j}$ and $\Delta_{T_{j}} f_{j}=0$ is immediate. Let us consider the cases of $j=2, \ldots, k$. Then the two transformations $T=T_{1}$ and $S=T_{j}$ are related. So now let $T_{j}^{n_{j}}=T_{1}^{m}$ for some $m \in \mathbb{N}$ and $n_{j} \in \mathbb{Z} \backslash\{0\}$. Let us now take $k_{j}:=\min \left\{\ell \in \mathbb{N}: \exists \nu \in \mathbb{Z} \backslash\{0\} T_{j}^{\nu}=T_{1}^{\ell}\right\}, j=2, \ldots, k$. Clearly, we have then $k_{j} \mid m$ and $k_{j} \mid m_{1}$. From this and the $T_{j}$-periodicity of $g_{j}$ we obtain

$$
\frac{1}{m} \sum_{\mu=0}^{m-1} g_{j}\left(T_{1}^{\mu}(x)\right)=\frac{1}{k_{j}} \sum_{\mu=0}^{k_{j}-1} g_{j}\left(T_{1}^{\mu}(x)\right)=\frac{1}{m_{1}} \sum_{\mu=0}^{m_{1}-1} g_{j}\left(T_{1}^{\mu}(x)\right)=G_{j}(x) .
$$

Therefore, also using $\Delta_{T_{1}} G_{j}(x)=0$ for $j=2, \ldots, k$, we get

$$
\sum_{\mu=0}^{m-1} F_{j}\left(T_{1}^{\mu}(x)\right)=\sum_{\mu=0}^{m-1} g_{j}\left(T_{1}^{\mu}(x)\right)-\sum_{\mu=0}^{m-1} G_{j}\left(T_{1}^{\mu}(x)\right)=m \cdot G_{j}(x)-\sum_{\mu=0}^{m-1} G_{j}(x)=0 .
$$

This shows that for $T=T_{j}$ and $S=T_{1}$ the assumptions of Lemma 3.3 are satisfied; hence the application of this lemma furnishes $T_{j}$-periodic functions $f_{j}: A \rightarrow \mathbb{R}$ with $\Delta_{T_{1}} f_{j}=F_{j}, j=2, \ldots, k$.

Finally, we set $f_{1}:=f-\left(f_{2}+f_{3}+\cdots+f_{n}\right)$. Using (8) we see that

$\Delta_{T_{1}} f_{1}=\Delta_{T_{1}} f-\left(\Delta_{T_{1}} f_{2}+\Delta_{T_{1}} f_{3}+\cdots+\Delta_{T_{1}} f_{n}\right)=\Delta_{T_{1}} f-\left(F_{2}+F_{3}+\cdots+F_{n}\right)=0$.

Thus $f=f_{1}+f_{2}+\cdots+f_{n}$ is a desired periodic decomposition of $f$.

\section{Generalizations and applications}

We start this section by proving that Corollary 2.7 also holds for $\Gamma$-valued functions for an arbitrary torsion free Abelian group $\Gamma$.

Theorem 4.1. Let $\mathcal{A}, \Gamma$ be torsion free Abelian groups and $a_{1}, \ldots, a_{n} \in \mathcal{A}$. $A$ function $f: \mathcal{A} \rightarrow \Gamma$ decomposes into a sum of $a_{j}$-periodic functions $f_{j}: \mathcal{A} \rightarrow \Gamma$, $f=f_{1}+\cdots+f_{n}$, if and only if for all partitions $B_{1} \cup B_{2} \cup \cdots \cup B_{N}=\left\{a_{1}, \ldots, a_{n}\right\}$ and $b_{j}$ being the least common multiple of the elements in $B_{j}$ one has

$$
\Delta_{b_{1}} \ldots \Delta_{b_{N}} f=0
$$


Proof. Since the proof of the necessity worked for arbitrary $\mathcal{A}, \Gamma$, it suffices to prove sufficiency. This we prove by induction on $n$, the case $n=1$ being obvious. So let $n>1$ and let us assume that the assertion holds for any smaller number of translates.

We can (re)number the translates $\left\{a_{2}, \ldots, a_{n}\right\}$ so that $a_{2}, \ldots, a_{k}$ are exactly the elements that have a common multiple with $a_{1}$. Let $b$ denote the least common multiple of $a_{1}, \ldots, a_{k}$ and take the integers $m_{1}, \ldots, m_{k}$ such that $b=m_{1} a_{1}=\cdots=$ $m_{k} a_{k}$. Using that $\mathcal{A}$ is torsion free, it is easily seen that $m_{1}, \ldots, m_{k}$ are relatively prime and so there exist integers $d_{1}, \ldots, d_{k}$ for which $d_{1} m_{1}+\cdots+d_{k} m_{k}=1$. We can suppose that $\Gamma \leq \mathbb{Q}^{I}$, since a torsion free Abelian group can be embedded as a subgroup in $\mathbb{Q}^{I}$ for some $I$. As we saw in Remark 3.4.2, for a function $f$ with values in $\Gamma \leq \mathbb{Q}^{I}$ the proof of Theorem 2.3 gives only a decomposition with values in $\frac{1}{M} \Gamma$ where $M=M\left(T_{1}, T_{2}, \ldots, T_{n}\right)$ is a certain positive integer. Furthermore, by Remark 3.4.3, the constant $m_{1} M\left(T_{2}, \ldots, T_{n}\right) M\left(T_{k+1}, \ldots, T_{n}\right)$ is an appropriate choice for $M\left(T_{1}, T_{2}, \ldots, T_{n}\right)$. By the induction hypothesis, however, we can already assume that $M\left(T_{2}, \ldots, T_{n}\right)=M\left(T_{k+1}, \ldots, T_{n}\right)=1$, so we get a decomposition $f=f_{1}^{1}+$ $\cdots+f_{n}^{1}$ where $f_{j}^{1}: \mathcal{A} \rightarrow \frac{1}{m_{1}} \Gamma$ is an $a_{j}$-periodic function $(j=1, \ldots, n)$. Repeating the same argument for $a_{i}(1 \leq i \leq k)$ instead of $a_{1}$, we obtain $k$ decompositions of $f$ with the following properties:

$$
f=f_{1}^{i}+f_{2}^{i}+\cdots+f_{n}^{i}, \text { where } f_{j}^{i}: \mathcal{A} \rightarrow \frac{1}{m_{i}} \Gamma \text { is } a_{j} \text {-periodic }(i=1, \ldots, k) .
$$

Take the linear combination of the decompositions $f_{j}^{i}$ with coefficients $d_{i} m_{i}$ :

$$
f_{j}:=\sum_{i=1}^{k} d_{i} m_{i} f_{j}^{i}
$$

Being the linear combination of $a_{j}$-periodic functions, $f_{j}$ is $a_{j}$-periodic, too. Moreover, $f_{j}$ is $\Gamma$-valued because the functions $m_{i} f_{j}^{i}$ are all $\Gamma$-valued $(i=1, \ldots, k)$. Finally, the functions $f_{j}$ indeed give a suitable decomposition of $f$, because

$$
\sum_{j=1}^{n} f_{j}=\sum_{j=1}^{n} \sum_{i=1}^{k} d_{i} m_{i} f_{j}^{i}=\sum_{i=1}^{k} d_{i} m_{i} \sum_{j=1}^{n} f_{j}^{i}=\left(\sum_{i=1}^{k} d_{i} m_{i}\right) \cdot f=f .
$$

Let $\mathcal{A}$ be a torsion free Abelian group. Using the above theorem for $\Gamma=\mathbb{R}$ and then for $\Gamma=\mathbb{Z}$, we get that for a function $f: \mathcal{A} \rightarrow \mathbb{Z}$ the existence of a real-valued periodic decomposition and the existence of an integer-valued periodic decomposition are both equivalent with the same condition. Thus we obtain the following.

Corollary 4.2. If an integer-valued function $f$ on a torsion free Abelian group $\mathcal{A}$ decomposes into the sum of $a_{j}$-periodic real-valued functions, $f=f_{1}+f_{2}+\cdots+f_{n}$ for some $a_{1}, \ldots, a_{n}$, then $f$ also decomposes into the sum of $a_{j}$-periodic integervalued functions, $f=g_{1}+g_{2}+\cdots+g_{n}$.

The following example shows that neither Theorem 4.1 nor Corollary 4.2 holds for every Abelian group $\mathcal{A}$. For instance let $\mathcal{A}$ be $\mathbb{Z}_{2} \times \mathbb{Z}_{2}$ and $a_{1}=(1,0) ; a_{2}=(0,1)$; $a_{3}=(1,1) \in \mathbb{Z}_{2} \times \mathbb{Z}_{2}$. We can represent functions $f: \mathbb{Z}_{2} \times \mathbb{Z}_{2} \rightarrow \mathbb{R}$ by $2 \times 2$ real matrices: $\left(\begin{array}{ll}f(0,0) & f(0,1) \\ f(1,0) & f(1,1)\end{array}\right)$. Let us consider the following decomposition:

$$
\left(\begin{array}{ll}
0 & 1 \\
1 & 1
\end{array}\right)=\left(\begin{array}{cc}
0 & \frac{1}{2} \\
0 & \frac{1}{2}
\end{array}\right)+\left(\begin{array}{cc}
0 & 0 \\
\frac{1}{2} & \frac{1}{2}
\end{array}\right)+\left(\begin{array}{cc}
0 & \frac{1}{2} \\
\frac{1}{2} & 0
\end{array}\right)
$$


The functions $f_{j}: \mathbb{Z}_{2} \times \mathbb{Z}_{2} \rightarrow \mathbb{R}$ represented by the matrices on the right are clearly $a_{j}$-periodic respectively for $j=1,2,3$. However, there does not exist an integervalued decomposition since the sum of the entries of a matrix that represents an $a_{j}$-periodic integer-valued function $g_{j}: \mathbb{Z}_{2} \times \mathbb{Z}_{2} \rightarrow \mathbb{Z}$ must be even $(j=1,2,3)$. Also the sums of such matrices do have this property, whereas $\left(\begin{array}{ll}0 & 1 \\ 1 & 1\end{array}\right)$ does not.

Finally, for measurable decompositions we can draw the following consequence of our results. Proposition 3.3 in [8] tells us that the equivalence of i) and ii) in Corollary 4.3 below is valid, whenever the statement of Corollary 4.2 is true, what we already have proved. This consideration yields the following.

Corollary 4.3. The following two assertions are equivalent for arbitrary real numbers $a_{1}, \ldots, a_{n}$.

i) If an integer-valued function $f: \mathbb{R} \rightarrow \mathbb{Z}$ can be decomposed as $f=f_{1}+\cdots+f_{n}$ such that each $f_{j}$ is an $a_{j}$-periodic measurable $\mathbb{R} \rightarrow \mathbb{R}$ function, then $f$ can also be decomposed as $f=g_{1}+\cdots+g_{n}$ such that each $g_{j}$ is an $a_{j}$-periodic integer-valued measurable function.

ii) If $B_{1}, \ldots, B_{N}$ are the equivalence classes of $\left\{a_{1}, \ldots, a_{n}\right\}$ with respect to the relation $a \sim b \Leftrightarrow a / b \in \mathbb{Q}$, and $b_{j}$ denotes the least common multiple of the numbers in $B_{j}(j=1, \ldots, N)$, then $\frac{1}{b_{1}}, \ldots, \frac{1}{b_{N}}$ are linearly independent over $\mathbb{Q}$.

\section{REFERENCES}

[1] B. Farkas, Sz.Gy. Révész, Decomposition as the sum of invariant functions with respect to commuting transformations, Aequationes Math. 73 (2007), 233-248. MR2327337

[2] Z. Gajda, Note on decomposition of bounded functions into the sum of periodic terms, Acta Math. Hung. 59 (1992), no. 1-2, 103-106. MR1160205 (93d:39001)

[3] V.M. Kadets, S.B. Shumyatskiy, Averaging Technique in the Periodic Decomposition Problem, Mat. Fiz. Anal. Geom. 7 (2000), no. 2, 184-195. MR1788194 (2001f:46022)

[4] V.M. Kadets, S.B. Shumyatskiy, Additions to the Periodic Decomposition Theorem, Acta Math. Hungar. 90 (2001), no. 4, 293-305. MR1910714 (2003g:46010)

[5] T. Keleti, Difference functions of periodic measurable functions, Ph.D. dissertation, ELTE, Budapest, 1996.

[6] T. Keleti, On the differences and sums of periodic measurable functions, Acta Math. Hungar. 75 (1997), no. 4, 279-286. MR1448704 (98e:26007)

[7] T. Keleti, Difference functions of periodic measurable functions, Fund. Math. 157 (1998), 15-32. MR1619289 (99e:28007)

[8] T. Keleti, Periodic decomposition of measurable integer valued functions, J. Math. Anal. Appl., to appear.

[9] Gy. Károlyi, T. Keleti, G. Kós, I.Z. Ruzsa, Periodic decomposition of integer valued functions, Acta Math. Hungar., to appear.

[10] M. Laczkovich, Sz.Gy. Révész, Periodic decompositions of continuous functions, Acta Math. Hungar. 54 (1989), no. 3-4, 329-341. MR1029096 (90k:26006)

[11] M. Laczkovich, Sz.Gy. Révész, Decompositions into periodic functions belonging to a given Banach space, Acta Math. Hung. 55(3-4) (1990), 353-363. MR1091818 (92a:39003)

[12] S. Mortola, R. Peirone, The sum of periodic functions, Boll. Un. Mat. Ital. 8 2-B (1999), 393-396. MR1706564 (2000e:26007)

[13] M. Wierdl, Continuous functions that can be represented as the sum of finitely many periodic functions, Mat. Lapok 32 (1984), 107-113 (in Hungarian). MR839598 
Technische Universität Darmstadt, Fachbereich Mathematik, AG4, SchlossgartenStrasse 7, D-64289, Darmstadt, Germany

E-mail address: farkas@mathematik.tu-darmstadt.de

Department of Analysis, Eötvös Loránd University, Pázmány Péter sétány 1/C, H1117 Budapest, Hungary

E-mail address: bizkit@cs.elte.hu

Department of Analysis, Eötvös Loránd University, Pázmány Péter sétány 1/C, H1117 BudAPEST, Hungary

E-mail address: elek@cs.elte.hu

A. Rényi Institute of Mathematics, Hungarian Academy of Sciences, Budapest, P.O.B. 127, 1364 HungarY

E-mail address: revesz@renyi.hu 